\title{
H. pylori infection is related to mitochondrial microsatellite instability in gastric carcinogenesis
}

Xianlong Ling, Haoxiang Zhang, Caifei Shen, Wu Yan, Pu Wang, Ji Feng, Zhihong Peng, Guiyong Peng, Wensheng Chen and Dianchun Fang ${ }^{*}$

\begin{abstract}
Bachground: To assess the correlation of H. pylori infection with mitochondrial microsatellite instability (mtMSI) and IL-8 in gastric carcinogenesis.

Methods: $H$. pylori infection was evaluated through histology and a urease breath test; mtMSI was measured using PCR-single strand conformation polymorphism (PCR-SSCP); IL-8 was analyzed with ELISA methods.

Results: The detection rate of mtMSI was significantly higher in specimens with $H$. pylori infection than in those without $H$. pylori infection $(P<0.05)$. The levels of IL-8 were significantly higher in specimens with mtMSI than in those without mtMSI $(P<0.01)$.An association of mtMSI with the intestinal histological type was found $(P<0.05)$. Increased IL-8 levels induced by $H$. pylori were related to the invasion, lymphnode spreading and clinical stage of gastric cancer $(P<0.05)$.

Conclusions: $H$. pylori infection is related to mitochondrial microsatellite instability in the early steps of gastric cancer development. IL-8 may play a role in the development of mtMSI induced by H. pylori. Our results support a role for mtMSI in different mechanisms of gastric carcinogenesis.
\end{abstract}

Keywords: H. pylori, Mitochondrial microsatellite instability, IL-8, Gastric cancer

\section{Background}

Gastric carcinogenesis is a complex multistep multifactorial event, in which the role of Helicobacter pylori (H. pylori) infection, the major etiopathogenic factor underlying chronic antral gastritis and duodenal ulcer, has been established in recent years $[1,2]$. Infection with $H$. pylori, especially with CagA + strains, has been associated with an increased risk of noncardia gastric adenocarcinoma [3, 4]. The epidemiological link between $H$. pylori and gastric carcinoma has been confirmed, and the rate of gastric carcinoma development in the Japanese population with $H$. pylori-gastritis was reported to be $2.9 \%$ during a 7.8-year period [5]. The mechanism by which $H$. pylori contributes to gastric carcinogenesis is still largely unknown. The chronic inflammatory reaction caused by the bacterium is

\footnotetext{
* Correspondence: fangdianchun@hotmail.com

Department of Gastroenterology, Southwest Hospital, Third Military Medical University, Chongqing 400038, China
}

(c) 2016 The Author(s). Open Access This article is distributed under the terms of the Creative Commons Attribution 4.0 International License (http://creativecommons.org/licenses/by/4.0/), which permits unrestricted use, distribution, and reproduction in any medium, provided you give appropriate credit to the original author(s) and the source, provide a link to the Creative Commons license, and indicate if changes were made. The Creative Commons Public Domain Dedication waiver (http://creativecommons.org/publicdomain/zero/1.0/) applies to the data made available in this article, unless otherwise stated. directly involved in gastric carcinogenesis through its potential to cause excess production of reactive oxygen species and consequent mutagenic and carcinogenic changes in DNA $[6,7]$. ROS production has been shown to occur in association with this bacterium both in vitro and in vivo [8-10]. The dietary intake of antioxidants, measured as the total antioxidant potential, is inversely associated with the risk of both cardia and distal cancer [11].

$H$. pylori infection results in the induction of a number of genes in host cells that are potential determinants of inflammation. Interleukin-8 (IL-8), a CXC chemokine specific for neutrophil granulocyte chemotaxis, is a central mediator of the inflammatory response to $H$. pylori and has been found to be involved in $H$. pylori-associated gastric carcinogenesis [12]. IL-8 induction in gastric epithelial cells has been clearly correlated with a functional cagA gene [13]. In H. pylori strains that express CagA, cytokine expression has been linked to an 
elevated inflammatory response in vivo [14]. The expression of IL- 8 was found to be 10 times higher in gastric cancer tissue than in normal tissue and to be two times higher in advanced gastric cancer tissue than early cancer tissue [15]. The eradication of $H$. pylori decreases IL-8 expression significantly, suggesting that gastric cancer may be associated with the inflammatory process in the gastric mucosa through the over-expression of IL-8 [16]. It is therefore expected that intervention with an IL-8 inhibitor would inhibit or reverse the process of $H$. pylori-related carcinogenesis.

Genetic instability plays a key role in neoplastic transformation and progression [17, 18]. A previous study by our group showed that in gastric carcinomas, such genetic instability may be classified into two different forms, in which hypermutability occurs by means of either chromosomal instability or microsatellite instability (MSI) $[19,20]$. Although a wide spectrum of MSI alterations in the nuclear DNA (nMSI) of cancer cells has been established, little attention has been paid to MSI in the mitochondrial DNA (mtMSI) of $H$. pylori-infected gastric tissues. The mitochondrial genome is more vulnerable to oxidative damage and experiences a higher rate of mutation than the nuclear genome [21]. The biology of the mitochondrion suggests that its genome may be an attractive target for ROS, which could drive tumorigenesis. Tumor formation is often associated with mtDNA mutations and alterations in mitochondrial genome function. Our previous studies have identified sequential accumulation of mtMSI in the histological progression from chronic gastritis to gastric cancer, suggesting that mtMSI may play an early and important role in the gastric carcinogenesis pathway, especially in intestinal-type cancer and distal gastric cancer [22]. However, the role of mtMSI following oxidative DNA damage in $H$. pylori-related gastric carcinoma is less clear. In the present study, we explored the associations between $H$. pylori infection, mtMSI and IL-8 to elucidate whether mtMSI triggers the progression from $H$. pylorigastritis to intestinal metaplasia and dysplasia and, finally, to gastric cancer.

\section{Methods}

\section{Tissue samples}

A total of 122 fresh samples from surgically resected gastric carcinomas and 100 fresh samples of endoscopically obtained non-neoplastic gastric mucosa (40 chronic gastritis, 40 intestinal metaplasia and 20 dysplasia mucosa) were studied. In addition, we collected 30 gastric biopsies from patients without any gastric disease as a control for the analysis of mtMSI and IL-8 alterations. Hematoxylin-eosin (HE) staining was performed for histopathological diagnosis and to evaluate gastritis, atrophy, intestinal metaplasia, dysplasia and cancer.
Genomic DNA was isolated using standard proteinase K digestion and phenol-chloroform extraction protocols. No patient had received non-steroidal anti-inflammatory drugs, proton pump inhibitors, or antibiotics within the previous three months. None of the gastric cancer patients included in the present series had a family history suggestive of HNPCC or had received chemotherapy or radiation therapy. This study was approved by the Bioethical Committee of the southwest hospital, and all patients signed informed consent forms before inclusion.

\section{Histology}

The surgically resected and biopsy specimens were fixed in $10 \%$ buffered formalin, embedded in paraffin wax, cut into sequential $4 \mu \mathrm{m}$ sections, and subjected to hematoxylin and eosin (H\&E) and modified Giemsa staining. In most cases, we cut three to five serial sections from each specimen, and multiple high power fields were examined. Virtually all of the specimens included the surface epithelium and muscularis mucosa. The specimens were examined by one experienced histologist blinded to the patient's clinical diagnosis and $H$. pylori characteristics.

\section{H. pylori assessment}

The presence of $H$. pylori was determined through histology and a urease breath test. Patients were classified as H. pylori-positive if at least one of the examinations produced a positive result.

\section{IL-8 quantification}

For IL-8 protein quantification, we used the same biopsy specimens as were used for $H$. pylori detection. Biopsy specimens were placed in $1.5 \mathrm{ml}$ of $\mathrm{PBS}(\mathrm{pH} 7.4)$ and were immediately homogenized using a tissue homogenizer, as described previously [23]. Aliquots of the homogenate supernatant, obtained via centrifugation $(10,000 g$ for $10 \mathrm{~min}$ ), were stored at $-80{ }^{\circ} \mathrm{C}$ until the assessment of total proteins using a modified Lowry method. IL-8 in the biopsy homogenate supernatant was measured via ELISA using commercially available assay kits (Research and Diagnostic Systems, Minneapolis, Minnesota, USA). The assays were performed in duplicate according to the manufacturer's instructions. In our laboratory, the ELISA's sensitivity for IL-8 was approximately $10 \mathrm{pg} / \mathrm{ml}$.

\section{mtMSI detection}

PCR-single-strand conformation polymorphism (PCRSSCP) analysis was performed to amplify mtDNA microsatellite sequences using published primers [24]. The primers consisted of 2 D-loop regions and 5 coding regions (Table 1). The reaction conditions and procedures were similar to those reported by Hebano et al. [24]. 
Table 1 Primer sequences for PCR analysis

\begin{tabular}{|c|c|c|c|c|}
\hline Repeat sequence & mtDNA region & Position & Annealing $\left({ }^{\circ} \mathrm{C}\right)$ & $\operatorname{Primer}\left(5^{\prime}-3^{\prime}\right)$ \\
\hline \multirow[t]{2}{*}{$\overline{(C) n}$} & $270-425$ & D-loop & 58 & TCCACACAGACATCAATAACA \\
\hline & & & & AAAGTGCATACCGCCAAAAG \\
\hline \multirow[t]{2}{*}{$(C A) n$} & $467-556$ & D-loop & 55 & СCCATACTACTAATCTCATCAA \\
\hline & & & & TाTGGTTGGTTCGGGGTATG \\
\hline \multirow[t]{2}{*}{ (C) 6} & $3529-3617$ & ND1 & 55 & CCGACCTTAGCTCTCACCAT \\
\hline & & & & AATAGGAGGCCTAGGTTGAG \\
\hline \multirow[t]{2}{*}{ (A)7 } & $4555-4644$ & ND2 & 55 & CCTGAGTAGGCCTAGAAATAAA \\
\hline & & & & ACTTGATGGCAGCTTCTGTG \\
\hline \multirow[t]{2}{*}{ (T)7 } & $9431-9526$ & COIII & 55 & CCAAAAAGGCCTTCGATACG \\
\hline & & & & GCTAGGCTGGAGTGGTAAAA \\
\hline \multirow[t]{2}{*}{ (C) 6 and(A) 8} & $12360-12465$ & ND5 & 55 & САCCCTAACCCTGACTTCC \\
\hline & & & & GGTGGATGCGACAATGGATT \\
\hline \multirow[t]{2}{*}{$(\mathrm{CCT}) 3$ and $(\mathrm{AGC}) 3$} & 12940-13032 & ND5 & 55 & GCCCTTCTAAACGCTAATCC \\
\hline & & & & TCAGGGGTGGAGACCTAATT \\
\hline
\end{tabular}

Each PCR product was digested with the appropriate restriction enzymes and electrophoresed at $300 \mathrm{~V}$ at $22{ }^{\circ} \mathrm{C}$ for $2 \mathrm{~h}$ in a $7.5 \%$ polyacrylamide gel containing $50 \mathrm{mmol} / \mathrm{L}$ boric acid, $1 \mathrm{mmol} / \mathrm{L}$ EDTA and $2.5 \%$ glycerol. After silver staining, PCR products that showed a mobility shift were defined as showing mtMSI. All analyses were repeated twice to rule out PCR artifacts.

\section{Statistical analysis}

The mucosal level of the IL- 8 protein was expressed in $\mathrm{pg} / \mathrm{mg}$ biopsy protein, and the data were expressed as the median and range. Wilcoxon's matched pairs test was used to compare differences in IL- 8 protein expression between $H$. pylori-positive and $H$. pylorinegative specimens. The relationship between $H$. pylori and the production of IL- 8 and mtMSI was assessed using Spearman's rank correlation coefficient and the Chi-square test with Yates' correction. A $P$ value of less than 0.05 was accepted as statistically significant.

\section{Results}

\section{H. pylori infection in different gastric diseases}

As shown in Table 2, the rate of $H$. pylori infection was $45.0 \%$ in chronic gastritis, $55.0 \%$ in intestinal metaplasia, $45.0 \%$ in dysplasia and $42.6 \%$ in gastric cancer. No $H$. pylori was detected in normal gastric mucosae. The rates of $H$. pylori infection in chronic gastritis, intestinal metaplasia, dysplasia and gastric cancer were significantly higher than in the normal gastric mucosa.

\section{H. pylori infection and IL-8 expression}

The IL-8 expression levels associated with different gastric pathologies are shown in Fig. 1. H. pylori infection was associated with increased expression of IL-8; the level of IL-8 was significantly higher in various gastric mucosae with $H$. pylori infection than in those without H. pylori infection $(P<0.01)$.

\section{H. pylori infection and mtMSI}

All tumor and non-neoplastic gastric mucosal samples were screened for mtMSI at seven repeat sites using the PCR-RFLP method. Fig. 2a shows a representative mobility-shift band compared with normal counterparts. The rates of mtMSI in different gastric pathologies are shown in Fig. 2b. H. pylori infection was associated with increased mtMSI in various gastric pathologies, and the rate of mtMSI was significantly higher in specimens with $H$. pylori infection than in those without $H$. pylori infection $(P<0.01)$.

\section{Expression of IL-8 in gastric mucosae with mtMSI}

The relationship between IL- 8 expression and mtMSI is shown in Fig. 3. The levels of IL-8 were significantly higher in gastric mucosae with mtMSI than in those without mtMSI $(P<0.05)$.

Table 2 H. pylori infection in different gastric diseases

\begin{tabular}{llll}
\hline & $n$ & H. pylori+ & H. pylori - \\
\hline Normal mucosa & 30 & 0 & 30 \\
Chronic gastritis & 40 & $18(45.0)$ & $22(65.0)$ \\
Intestinal metaplasia & 40 & $22(55.0)$ & $18(45.0)$ \\
Dysplasia & 20 & $9(45.0)$ & $11(55.0)$ \\
Gastric cancer & 122 & $52(42.6)$ & $70(57.4)$ \\
\hline
\end{tabular}




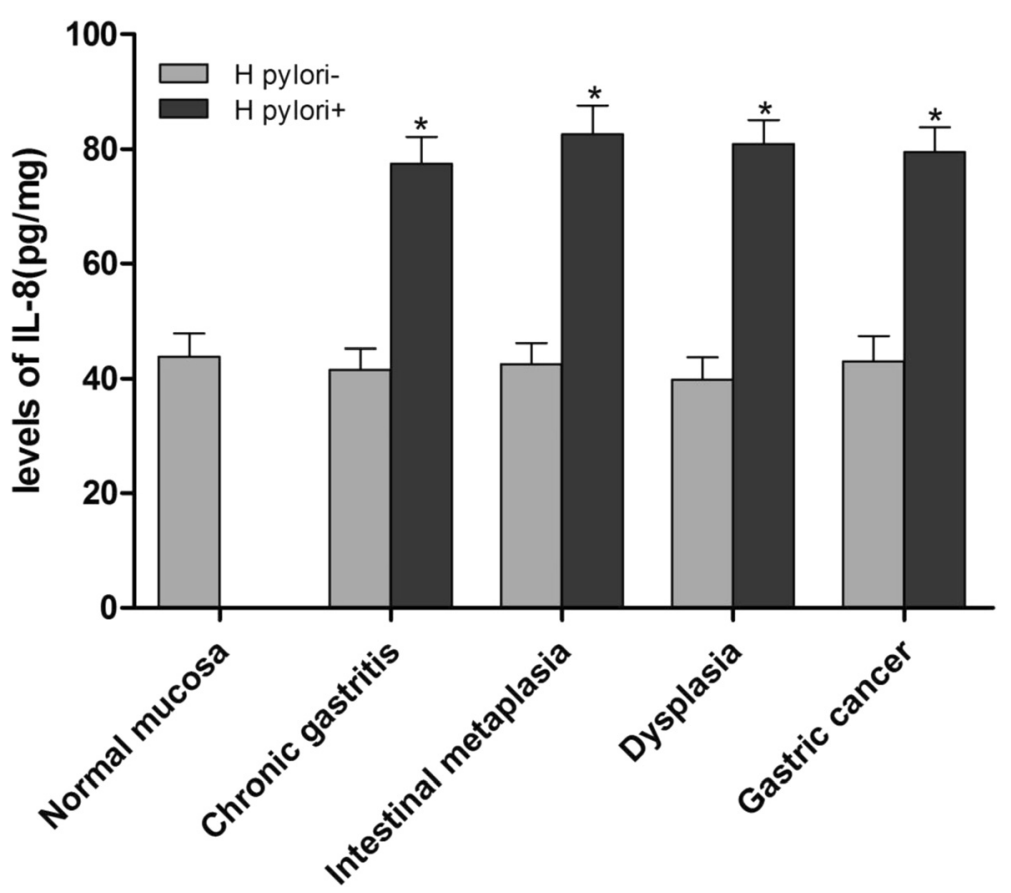

Fig. 1 The relationship between $H$. pylori infection and IL-8 expression. The levels of IL-8 were significantly higher in various gastric mucosae with H. pylori infection than in those without H. pylori infection $\left({ }^{*} P<0.01\right)$

\section{Relationship between IL-8 levels and clinicopathological} characteristics of gastric cancer

The relationships between IL- 8 levels and the clinicopathological characteristics of gastric cancer are shown in Table 3. Associations between IL-8 levels and invasion, lymph node spreading and clinical staging were found $(P<0.01)$, whereas no significant correlation was found between IL- 8 levels and the age at diagnosis, sex or tumor size $(P>0.05)$.

\section{Clinicopathological characteristics of mtMSI-positive gastric cancer}

The clinicopathological characteristics of mtMSI-positive cases were compared with those of cases that were mtMSI negative (Table 4). Associations of mtMSI with the intestinal histological type and a distal location were found $(P<0.05)$, whereas no significant correlation was found between mtMSI and the age at diagnosis, sex, tumor size, tumor location, invasion, lymph node spread or clinical stage $(P>0.05)$.

\section{Discussion}

Although a causal relationship exists between $H$. pylori infection and the development of gastric carcinoma, the molecular mechanisms underlying this relationship have remained elusive. In this study, we show that $H$. pylori infection may correlate with up-regulated IL-8 expression and mtMSI in the gastric mucosa of patients with various gastric pathologies. Up-regulation of IL-8 is correlated with
mtMSI. These findings are consistent with IL-8 and mtMSI playing important roles in $H$. pylori-associated gastric cancer. Taking into consideration the increase in the mtMSI frequency observed in $H$. pylori-associated lesions, our results suggest early involvement and continuous accumulation of mtMSI in gastric cells with $H$. pylori infection that have entered the multistep gastric carcinogenesis pathway.

Mutations in the mitochondrial genome have been detected in nearly every type of cancer investigated to date [25], including gastric cancer and the corresponding preneoplastic lesions [26]. H. pylori infection has been reported as an important risk factor for gastric carcinogenesis [5, 27]. Previous studies have identified a significant association of $H$. pylori infection and gastric cancer with nMSI [24, 28]. Although some studies addressing mitochondrial mutations in gastric cancer have attempted to identify the role of mitochondrial genetic instability $[22,24,29]$, reports on the role of $H$. pylori in mtMSI in gastric carcinogenesis have been rare. mtDNA mutations are associated with $H$. pylori infections responsible for chronic gastritis and peptic ulcer tissues, indicating that the consequences of $H$. pylori infection include the aggregation of mutations in mtDNA in early phases of gastric cancer development. Machado et al. [30] showed that gastric epithelial cells are closely related to the genetic instability of mitochondrial DNA due to $H$. pylori infection. To study the role of mtMSI in $H$. pylori-associated gastric carcinogenesis, we analyzed mtMSI in $H$. pylori-positive and $H$. pylori-negative 

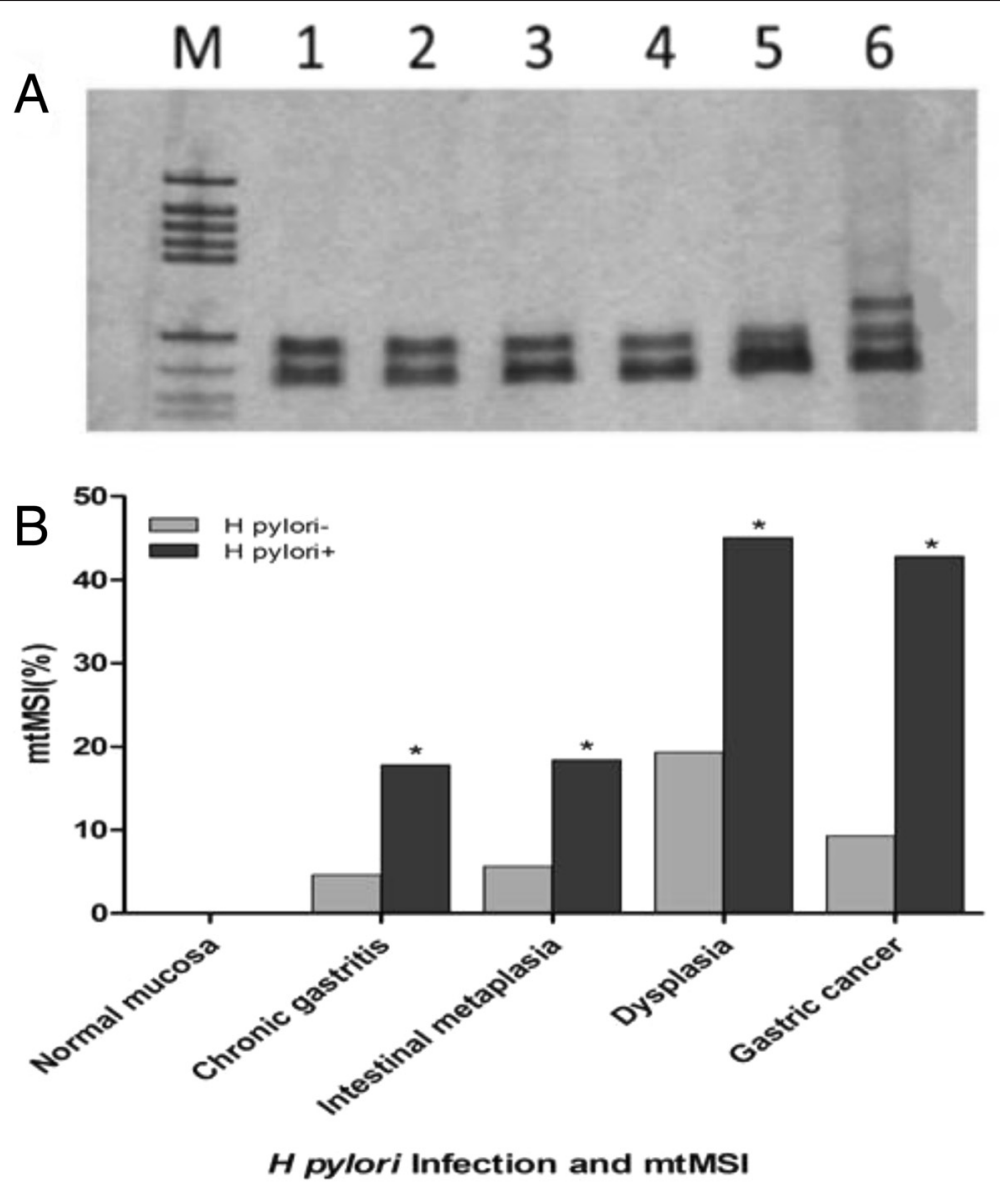

Fig. 2 The relationship between $H$. pylori infection and mtMSI. a. Detection of mtMSI via PCR-single strand conformation polymorphism analysis. Lane 6 shows conformational variants associated with mtMSI. b. The detection rate of mtMSI was significantly higher in specimens with $H$. pylori infection than in those without H. pylori infection $\left({ }^{*} P<0.05\right)$

gastric mucosa using seven microsatellite markers known to be altered in gastrointestinal carcinomas. It was found that as carcinogenesis progresses, the level of mtMSI increases, and the rate of mtMSI was found to be significantly higher in the $H$. pylori-positive groups than the $H$. pylori-negative groups, implying that mtMSI might play a role in the occurrence of those gastric cancers that are $H$. pylori positive.

The mechanisms underlying mtMSI induced by $H$. pylori in the gastric mucosa remain unclear. Reactive oxygen species (ROS) are commonly released in gastric mucosae that are inflamed as a result of infection with H. pylori, especially with CagA+ strains, and could be responsible for mtMSI-positive gastric cancer [31, 32]. The mitochondrial genome is particularly susceptible to oxidative damage and mutation because of the high rate of ROS generation in this organelle and its inefficient DNA repair system [33, 34]. Increased damage caused by ROS and defective DNA repair are the two causes that have been proposed to explain mtMSI in $H$. pylori-associated gastric cancer [31, 33]. A significant correlation between high IL-8 expression in the gastric mucosa and gastric cancer risk has been reported [35]. In IL-8 transgenic mice, the expression of IL- 8 increases tumorigenesis, suggesting that IL-8 might play a crucial role in gastrointestinal cancers [36]. The induction of higher levels IL-8 expression by $H$. pylori has been observed in gastric carcinoma and premalignant lesions [12]. In the present study, we found that the levels of IL- 8 were significantly higher in patients with mtMSI than in those without mtMSI, suggesting that IL-8 may play a role in the development of mtMSI induced by $H$. pylori. However, the mechanisms through which IL-8 leads to the development of mtMSI in $H$. pylori-positive gastric mucosae need to be studied further.

Cancers arising from different mutational pathways are thought to have different clinical features. nMSI+ gastric cancer is characterized by an older age, antral location, intestinal type, lower prevalence of lymph node metastasis, and a lower pTNM stage [37, 38]. However, the clinicopathological characteristics of mtMSI+ gastric cancers remain unclear. In the current 


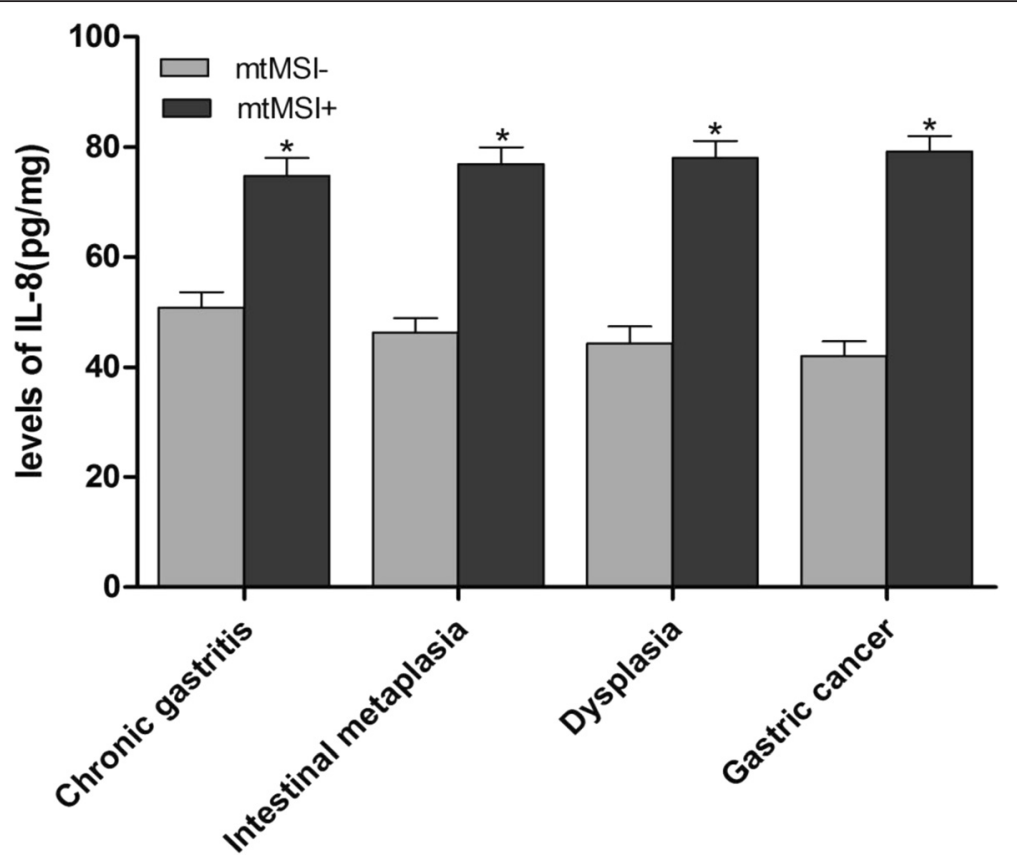

Fig. 3 L-8 levels in gastric mucosae with mtMSI. The levels of IL-8 were significantly higher in gastric mucosae with mtMSI than in those without mtMSI $\left({ }^{*} P<0.05\right)$

Table 3 Relationships between IL-8 levels and clinicopathological characteristics of gastric cancer

\begin{tabular}{llll}
\hline Characteristic & & $n$ & level of IL-8 \\
\hline Sex & Male & 88 & $62.2 \pm 3.1$ \\
& Female & 34 & $58.2 \pm 3.2$ \\
Age & $<40$ years & 39 & $58.2 \pm 3.6$ \\
& $>40$ years & 83 & $61.2 \pm 3.4$ \\
Size & $<5 \mathrm{~cm}$ & 62 & $57.2 \pm 3.5$ \\
& $>5 \mathrm{~cm}$ & 60 & $63.2 \pm 2.9$
\end{tabular}

Histological type

$\begin{array}{lll}\text { Intestinal } & 72 & 64.2 \pm 3.3 \\ \text { Diffuse } & 50 & 56.2 \pm 3.9\end{array}$

Tumor location

$\begin{array}{lll}\text { Distal } & 81 & 57.2 \pm 2.8 \\ \text { Proximal } & 41 & 63.2 \pm 3.5\end{array}$

Invasion

$\begin{array}{lll}\text { Within the wall } & 62 & 48.2 \pm 3.1^{*} \\ \text { Invading serosa } & 60 & 72.2 \pm 2.7\end{array}$

Lymph node spread

$\begin{array}{lll}\text { Absent } & 58 & 35.2 \pm 2.6^{*} \\ \text { Present } & 64 & 76.2 \pm 3.1\end{array}$

TNM stage

$\begin{array}{lll}\text { I/II } & 53 & 38.4 \pm 3.2^{*} \\ \text { III/IV } & 69 & 74.5 \pm 2.9\end{array}$

${ }^{*} P<0.01$ vs. the invading serosa, lymph node spread and stage III/IV groups study, we did not find an obvious relationship between mtMSI and tumor size, the depth of invasion, node metastasis or clinical stage, indicating a limited role of mtMSI in predicting the prognosis of gastric carcinoma. However, a marked difference in mtMSI was noted in gastric cancers distinguished by histological type. mtMSI was significantly more frequent in intestinal-type gastric cancers than diffuse-type gastric cancers, suggesting that mtMSI is a predisposing event in intestinal gastric cancer.

IL-8 can regulate neovascularization, thereby promoting the growth and spread of human gastric carcinoma [39]. Yamaoka et al. found that the expression of IL-8 was 10 times higher in gastric cancer tissue than in normal tissue and it was twice as high in advanced gastric cancer tissue compared with early cancer tissue [15]. Macrì et al. [40] reported that serum levels of IL-8 serve as a marker of gastric cancer. Increased expression of IL-8 mRNA in tissue extracts from gastric cancer patients has been associated with certain clinicopathological aspects of the disease, including a poor prognosis [41]. In a previous study, we showed that co-culture with H. pylori stimulates AGS cell motility and invasion, upregulates ezrin expression at the protein level and induces a Hummingbird phenotype [42]. In the present study, IL-8 levels were found to be associated with invasion, lymph node spreading and clinical stage. These observations indicate that high levels of IL- 8 may be associated with a poor prognosis and that IL- 8 may be indicative of more aggressive gastric cancer. 
Table 4 Clinicopathological characteristics of 122 gastric cancer patients with $\mathrm{mtMSI}$

\begin{tabular}{lllll}
\hline Characteristic & & $n$ & mtMSI+ & mtMSI- \\
\hline Age & $<40$ years & 39 & 9 & 30 \\
& $>40$ years & 83 & 19 & 64 \\
Sex & Male & 88 & 20 & 68 \\
Size & Female & 34 & 8 & 18 \\
Histological type & $<5 \mathrm{~cm}$ & 62 & 11 & 51 \\
& $>5 \mathrm{~cm}$ & 60 & 17 & 43 \\
Tumor location & Intestinal & 72 & $19^{*}$ & 53 \\
& Diffuse & 50 & 9 & 41 \\
Invasion & Distal & 81 & 21 & 60 \\
& Proximal & 41 & 7 & 34 \\
Lymph node spread & Within the wall & 62 & 18 & 44 \\
& Invading serosa & 60 & 10 & 50 \\
& Absent & 58 & 17 & 41 \\
& Present & 64 & 11 & 53
\end{tabular}

TNM stage

\begin{tabular}{llll} 
I/I & 53 & 13 & 40 \\
III/IV & 69 & 15 & 54 \\
\hline
\end{tabular}

${ }^{*} P<0.05$ vs. diffuse-type groups

\section{Conclusion}

In conclusion, we have demonstrated that mtMSI is an early and important event in the progression of gastric carcinogenesis, especially in intestinal-type gastric cancer. $H$. pylori infection contributes to mtMSI in early steps of gastric cancer development. IL-8 may play a role in the development of mtMSI induced by $H$. pylori. Our results support a role for mtMSI in different mechanisms of gastric carcinogenesis. Because the majority of patients with $H$. pylori infection will not progress to cancer and only a subset of these patients harbor mtMSI, it is conceivable that patients with $H$. pylori infection displaying mtMSI are at greater risk of developing gastric cancer than those without instability. As a surrogate marker for the risk of gastric cancer development, the role of mtMSI in gastric lesions with $H$. pylori infection warrants further investigation.

\section{Abbreviations}

H. pylori, Helicobacter pylori; HE, Hematoxylin-eosin; IL-8, Interleukin-8; mtMSI, mitochondrial microsatellite instability; PCR-SSCP, PCR-single strand conformation polymorphism.

\section{Acknowledgments}

This work was supported by the National Natural Science Foundation of China (No. 30070043).

\section{Authors' contributions}

$X L, H Z$ and CS participated in the design of the study, participated in the mtMSI detection and drafted the manuscript. WY and PW participated in the ELISA of IL-8. JF, ZP and GP collected the samples and participated in the $H$. pylori assessment.WC participated in the design of the study and performed the statistical analysis. DF conceived of the study, and participated in its design and coordination and helped to draft the manuscript. All authors read and approved the final manuscript.

\section{Competing interests}

The authors declare that they have no competing interests.

Received: 3 March 2016 Accepted: 18 May 2016

Published online: 12 July 2016

\section{References}

1. Graham DY. Helicobacter pylori update: gastric cancer, reliable therapy, and possible benefits. Gastroenterology. 2015;148:719-31.

2. el-Omar EM, Penman ID, Ardill JE, Chittajallu RS, Howie C, McColl KE. Helicobacter pylori infection and abnormalities of acid secretion in patients with duodenal ulcer disease. Gastroenterology. 1995;109:681-91.

3. Wu AH, Crabtree JE, Bernstein L, Hawtin P, Cockburn M, Tseng CC, et al. Role of Helicobacter pylori CagA+ strains and risk of adenocarcinoma of the stomach and esophagus. Int J Cancer. 2003;103:815-21.

4. Hansen S, Melby KK, Aase S, Jellum E, Vollset SE. Helicobacter pylori infection and risk of cardia cancer and non-cardia gastric cancer. A nested case-control study. Scand J Gastroenterol. 1999;34:353-60.

5. Uemura N, Okamoto S, Yamamoto S, Matsumura N, Yamaguchi S, Yamakido $M$, et al. Helicobacter pylori infection and the development of gastric cancer. N Engl J Med. 2001;345:784-9.

6. Drake IM, Mapstone NP, Schorah CJ, White KL, Chalmers DM, Dixon MF, et al. Reactive oxygen species activity and lipid peroxidation in Helicobacter pylori associated gastritis: relation to gastric mucosal ascorbic acid concentrations and effect of $\mathrm{H}$ pylori eradication. Gut. 1998:42:768-71.

7. Farinati F, Cardin R, Degan P, Rugge M, Mario FD, Bonvicini P, et al. Oxidative DNA damage accumulation in gastric carcinogenesis. Gut. 1998:42:351-6.

8. Davies GR, Simmonds NJ, Stevens TR, Sheaff MT, Banatvala N, Laurenson IF, et al. Helicobacter pylori stimulates antral mucosal reactive oxygen metabolite production in vivo. Gut. 1994;35:179-85.

9. Rautelin $\mathrm{H}$, Blomberg B, Fredlund $\mathrm{H}$, Järnerot $\mathrm{G}$, Danielsson D. Incidence of Helicobacter pylori strains activating neutrophils in patients with peptic ulcer disease. Gut. 1993:34:599-603.

10. Davies GR, Simmonds NJ, Stevens TR, Grandison A, Blake DR, Rampton DS. Mucosal reactive oxygen metabolite production in duodenal ulcer disease. Gut. 1992;33:1467-72.

11. Serafini M, Bellocco R, Wolk A, Ekström AM. Total antioxidant potential of fruit and vegetables and risk of gastric cancer. Gastroenterology. 2002;123:985-91.

12. Lee KE, Khoi PN, Xia Y, Park JS, Joo YE, Kim KK, et al. Helicobacter pylori and interleukin-8 in gastric cancer. World J Gastroenterol. 2013;19:8192-202.

13. Peek Jr RM, Miller GG, Tham KT, Perez-Perez Gl, Zhao X, Atherton JC, et al. Heightened inflammatory response and cytokine expression in vivo to cag $\mathrm{A}$ + Helicobacter pylori strains. Lab Invest. 1995;73:760-70.

14. Ando T, Kusugami K, Ohsuga M, Shinoda M, Sakakibara M, Saito H, et al, Interleukin-8 activity correlates with histological severity in Helicobacter pylori-associated antral gastritis. Am J Gastroenterol. 1996;91:1150-6.

15. Yamaoka Y, Kodama T, Kita M, Imanishi J, Kashima K, Graham DY. Relation between cytokines and Helicobacter pylori in gastric cancer. Helicobacter. 2001;6:116-24

16. Liao J, Wen $S$, Cao L, Zhou Y, Feng Z. Effect of eradication of Helicobacter pylori on expression Levels of FHIT, IL-8 and P73 in gastric mucosa of firstdegree relatives of gastric cancer patients. PLoS One. 2015;10, e0124576.

17. Lengauer C, Kinzler KW, Vogelstein B. Genetic instabilities in human cancers. Nature. 1998;396:643-9.

18. Ionov Y, Peinado MA, Malkhosyan S, Shibata D, Perucho M. Ubiquitous somatic mutations in simple repeated sequences reveal a new mechanism for colonic carcinogenesis. Nature. 1993:363:558-61.

19. Fang DC, Jass JR, Wang DX, Zhou XD, Luo YH, Young J. Infrequent loss of heterozygosity of APC/MCC and DCC genes in gastric cancer showing DNA microsatellite instability. J Clin Pathol. 1999;52:504-8.

20. Fang DC, Wang RQ, Yang SM, Yang JM, Liu HF, Peng GY, et al. Mutation and methylation of hMLH1 in gastric carcinomas with microsatellite instability. World J Gastroenterol. 2003;9:655-9.

21. Yakes FM, Van Houten B. Mitochondrial DNA damage is more extensive and persists longer than nuclear DNA damage in human cells following oxidative stress. Proc Natl Acad Sci U S A. 1997;94:514-9.

22. Ling $X L$, Fang $D C$, Wang $R Q$, Yang $S M$, Fang L. Mitochondrial microsatellite instability in gastric cancer and its precancerous lesions. World J Gastroenterol. 2004;10:800-3. 
23. Yamaoka Y, Kita M, Kodama T, Sawai N, Tanahashi T, Kashima K, et al. Chemokines in the gastric mucosa in Helicobacter pylori infection. Gut. 1998:42:609-17.

24. Habano W, Sugai T, Nakamura SI, Uesugi N, Yoshida T, Sasou S. Microsatellite instability and mutation of mitochondrial and nuclear DNA in gastric carcinoma. Gastroenterology. 2000;118:835-41.

25. Yu M. Somatic mitochondrial DNA mutations in human cancers. Adv Clin Chem. 2012;57:99-138.

26. Hiyama T, Tanaka S, Shima H, Kose K, Kitadai Y, Ito M, et al. Somatic mutation of mitochondrial DNA in Helicobacter pylori-associated chronic gastritis in patients with and without gastric cancer. Int J Mol Med. 2003;12:169-74.

27. Fukase K, Kato M, Kikuchi S, Inoue K, Uemura N, Okamoto S, et al. Effect of eradication of Helicobacter pylori on incidence of metachronous gastric carcinoma after endoscopic resection of early gastric cancer: an open-label, randomised controlled trial. Lancet. 2008;372:392-7.

28. Leung WK, Kim JJ, Kim JG, Graham DY, Sepulveda AR. Microsatellite instability in gastric intestinal metaplasia in patients with and without gastric cancer. Am J Pathol. 2000;156:537-43.

29. Jeong CW, Lee JH, Sohn SS, Ryu SW, Kim DK. Mitochondrial microsatellite instability in gastric cancer and gastric epithelial dysplasia as a precancerous lesion. Cancer Epidemiol. 2010:34:323-7.

30. Machado AM, Figueiredo C, Touati E, Máximo V, Sousa S, Michel V, et al. Helicobacter pylori infection induces genetic instability of nuclear and mitochondrial DNA in gastric cells. Clin Cancer Res. 2009;15:2995-3002.

31. Bagchi D, McGinn TR, Ye X, Bagchi M, Krohn RL, Chatterjee A, et al. Helicobacter pylori-induced oxidative stress and DNA damage in a primary culture of human gastric mucosal cells. Dig Dis Sci. 2002;47:1405-12.

32. Shimoyama T, Fukuda S, Liu Q, Nakaji S, Fukuda Y, Sugawara K. Production of chemokines and reactive oxygen species by human neutrophils stimulated by Helicobacter pylori. Helicobacter. 2002;7:170-4.

33. Akhmedov AT, Marín-García J. Mitochondrial DNA maintenance: an appraisal. Mol Cell Biochem. 2015:409:283-305.

34. Szczepanowska K, Trifunovic A. Different faces of mitochondrial DNA mutators. Biochim Biophys Acta. 1847:2015:1362-72.

35. Yamada S, Kato S, Matsuhisa T, Makonkawkeyoon L, Yoshida M, Chakrabandhu T, et al. Predominant mucosal IL-8 mRNA expression in noncagA Thais is risk for gastric cancer. World J Gastroenterol. 2013;19:2941-9.

36. Asfaha S, Dubeykovskiy AN, Tomita H, Yang X, Stokes S, Shibata W, et al. Mice that express human interleukin-8 have increased mobilization of immature myeloid cells, which exacerbates inflammation and accelerates colon carcinogenesis. Gastroenterology. 2013;144:155-66.

37. Wu MS, Lee CW, Shun CT, Wang HP, Lee WJ, Chang MC, et al. Distinct clinicopathologic and genetic profiles in sporadic gastric cancer with different mutator phenotypes. Genes Chromosomes Cancer. 2000;27:403-11.

38. Wu MS, Chang MC, Huang SP, Tseng CC, Sheu JC, Lin YW, et al. Correlation of histologic subtypes and replication error phenotype with comparative genomic hybridization in gastric cancer. Genes Chromosomes Cancer. 2001;30:80-6.

39. Kitadai $Y$, Haruma $K$, Sumii $K$, Yamamoto $S$, Ue T, Yokozaki $H$, et al. Expression of interleukin-8 correlates with vascularity in human gastric carcinomas. Am J Pathol. 1998;152:93-100.

40. Macrì A, Versaci A, Loddo S, Scuderi G, Travagliante M, Trimarchi G, et al. Serum levels of interleukin 1 beta, interleukin 8 and tumour necrosis factor alpha as markers of gastric cancer. Biomarkers. 2006;11:184-93.

41. Lee KH, Bae SH, Lee JL, Hyun MS, Kim SH, Song SK, et al. Relationship between urokinase-type plasminogen receptor, interleukin-8 gene expression and clinicopathological features in gastric cancer. Oncol. 2004:66:210-7.

42. Fan LL, Chen DF, Lan CH, Liu KY, Fang DC. Knockdown of ezrin via RNA interference suppresses Helicobacter pylori-enhanced invasion of gastric cancer cells. Cancer Biol Ther. 2011;11:746-52.

\section{Submit your next manuscript to BioMed Central and we will help you at every step:}

- We accept pre-submission inquiries

- Our selector tool helps you to find the most relevant journal

- We provide round the clock customer support

- Convenient online submission

- Thorough peer review

- Inclusion in PubMed and all major indexing services

- Maximum visibility for your research

Submit your manuscript at www.biomedcentral.com/submit
Biomed Central 